\title{
Genome differentiation in a species pair of coregonine fishes: an extremely rapid speciation driven by stress-activated retrotransposons mediating extensive ribosomal DNA multiplications
}

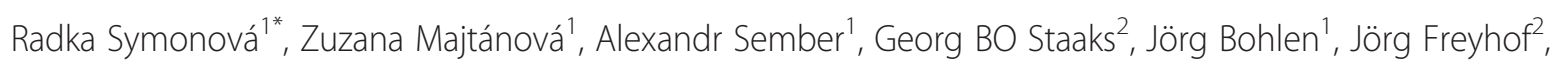
Marie Rábová ${ }^{1}$ and Petr Ráb ${ }^{1}$

\begin{abstract}
Background: Sympatric species pairs are particularly common in freshwater fishes associated with postglacial lakes in northern temperate environments. The nature of divergences between co-occurring sympatric species, factors contributing to reproductive isolation and modes of genome evolution is a much debated topic in evolutionary biology addressed by various experimental tools. To the best of our knowledge, nobody approached this field using molecular cytogenetics. We examined chromosomes and genomes of one postglacial species pair, sympatric European winter-spawning Coregonus albula and the local endemic dwarf-sized spring-spawning C. fontanae, both originating in Lake Stechlin. We have employed molecular cytogenetic tools to identify the genomic differences between the two species of the sympatric pair on the sub-chromosomal level of resolution.

Results: Fluorescence in situ hybridization (FISH) experiments consistently revealed a distinct variation in the copy number of loci of the major ribosomal DNA (the $45 S$ unit) between C. albula and C. fontanae genomes. In C. fontanae, up to 40 chromosomes were identified to bear a part of the major ribosomal DNA, while in C. albula only 8-10 chromosomes possessed these genes. To determine mechanisms how such extensive genome alternation might have arisen, a PCR screening for retrotransposons from genomic DNA of both species was performed. The amplified retrotransposon Rex1 was used as a probe for FISH mapping onto chromosomes of both species. These experiments showed a clear co-localization of the ribosomal DNA and the retrotransposon Rex 1 in a pericentromeric region of one or two acrocentric chromosomes in both species.

Conclusion: We demonstrated genomic consequences of a rapid ecological speciation on the level undetectable by neither sequence nor karyotype analysis. We provide indirect evidence that ribosomal DNA probably utilized the spreading mechanism of retrotransposons subsequently affecting recombination rates in both genomes, thus, leading to a rapid genome divergence. We attribute these extensive genome re-arrangements associated with speciation event to stress-induced retrotransposons (re)activation. Such causal interplay between genome differentiation, retrotransposons (re)activation and environmental conditions may become a topic to be explored in a broader genomic context in future evolutionary studies.
\end{abstract}

\footnotetext{
* Correspondence: radka.symonova@natur.cuni.cz

${ }^{1}$ Laboratory of Fish Genetics, Institute of Animal Physiology and Genetics,

Czech Academy of Sciences, Rumburská 89, Liběchov 277 21, Czech Republic

Full list of author information is available at the end of the article
}

\section{Biomed Central}

C 2013 Symonová et al.; licensee BioMed Central Ltd. This is an Open Access article distributed under the terms of the Creative Commons Attribution License (http://creativecommons.org/licenses/by/2.0), which permits unrestricted use, distribution, and reproduction in any medium, provided the original work is properly cited. 


\section{Background}

Intra-lacustrine fish speciation as an example of ecological speciation is a much debated topic in evolutionary biology addressed by various experimental tools, mostly in complex systems with a number of species, in particular in ancient freshwater lakes [1]. In Europe, with its comparatively depauperate fish fauna, issues of adaptive radiation and ecological speciation in fishes are highly relevant in temperate postglacial lakes (originating after the last glaciation i.e. 12-15 kyrs BP). To assess potential modes of speciation in fishes, numerous model systems are available [2], among which one of the best groups with a robust knowledge on adaptive speciation and complex speciation patterns in postglacial lakes are coregonine fishes (Coregoninae, [3]) [4-6]. Within coregonines, their numerous sympatric species pairs and recent species flocks [7-9] are of particular importance [10]. In Coregonus, based on extensive genetic and population genetic [11], phylogenetic, biogeographic, morphological and eco-physiological data, six potential modes of speciation have been proposed [12]. However, none of these approaches utilized cytogenetic data despite salmonid fishes, to which coregonines belong, being one of the best karyologically studied fish groups in terms of the number of species, populations, individuals and material (adults and embryos) examined. Available cytogenetic data demonstrate that salmonids include two basic karyotypes - the high chromosome number $2 \mathrm{n} \sim 80$ (type $\mathrm{A}$ and its derivatives) and the low chromosome number $2 \mathrm{n} \sim 60$ (type $\mathrm{B}$ and its derivatives) - co-occurring in all recognized salmonid phylogenetic lineages (except graylings, Thymallinae), including whitefish, ciscoes and innconu (Coregoninae). Species with the type B karyotypes have in common either prominent anadromous behaviour and/or are found in lacustrine environments and are likely products of intra-lacustrine speciation (for review [13]). Such apparent parallelism might be explained by specific life history strategies leading in both types of environments to small effective population sizes, thus, enabling increased probability of fixation of genic or chromosomal mutations. Observed evolution of chromosome number in salmonids is likely affected by selection for increased or decreased genetic recombination rate as proposed by Quimseyh [14], explaining high variability in chromosome numbers in mammals based on fundamental numbers (NF, chromosome arms number).

In this study, we examined chromosomes and genomes of the sympatric species Coregonus albula and C. fontanae in the dimictic Lake Stechlin, northern Germany to test whether the above outlined parallelism on karyotype differentiation in intralacustrine species pairs can also be observed in incipient speciation processes in young postglacial lakes. Both species are pelagic zooplanktivores, but they differ considerably in their size, spawning time
[15] and temperature-dependent metabolic physiological adaptations [16]. Up to now, C. fontanae has not yet been subjected to any cytogenetic analysis as opposed to C. albula (see [17] and references therein). The level of genetic differentiation between C. albula and C. fontanea tested by combined analyses of mitochondrial DNA and microsatellite loci showed a weak differentiation $\left(F_{\mathrm{ST}}=0-0.008\right)$ between these two species when compared with another sympatric species pair C. albula and C. lucinensis [18]. Further population genetic analyses based on 1244 polymorphic AFLP loci demonstrated a lower differentiation between allopatric than sympatric populations of the C. albula complex and suggested a rather complex colonization history than simple sympatric speciation [6]. Therefore, we have employed a novel approach in this field to explore the up to now neglected aspects of genome evolution in this species pair and used different parts of ribosomal DNA of the $45 \mathrm{~S}$ rDNA unit as cytotaxonomic markers.

At the first stage of this study, we have employed conventional methods of karyotype analysis (Giemsa and $\mathrm{Ag}$ staining, $\mathrm{CMA}_{3}$ and DAPI fluorescence). At the second stage, we have performed molecular cytogenetic analyses (CGH and FISH with various rDNA fragments and non-LTR retrotransposons as probes) to identify any differences between chromosomal complements of these two species on the sub-chromosomal level of resolution since the karyotype analyses showed no significant differences. At the third stage, we performed molecular biological analyses of the $45 \mathrm{~S}$ ribosomal RNA genes and the Rex1 non-LTR retrotransposon. Furthermore, we discuss these results in the context of populations of small effective sizes under extreme stress conditions under which retrotransposons (re)activation could have contributed to accelerated speciation. The major cluster of ribosomal RNA genes is expressed as the $45 \mathrm{~S}$ transcriptional unit (Figure 1). This unit consists of 18S, $5.8 \mathrm{~S}$ and $28 \mathrm{~S}$ rDNA genes, separated by internal transcribed spacers (ITS1, ITS2) and surrounded by external transcribed spacers (ETS). The $45 \mathrm{~S}$ rDNA units are arranged in tandem repetitions with high copy numbers $[19,20]$ therefore, they represent a useful cytotaxonomic marker. The individual units are separated by intergenic spacers (IGS) [21,22]. The structure and the order of genes within the unit are highly conserved among Eukaryota [23]. Different parts of the $45 \mathrm{~S}$ transcriptional unit display different mutational rates. The most conserved region is the 18S rRNA gene and the most variable are ITSs [23].

\section{Results}

\section{Karyotyping and comparative cytogenetics}

Karyotypes of both examined ciscoes were very similar $(2 \mathrm{n}=80$ in both species) and both belong to the 


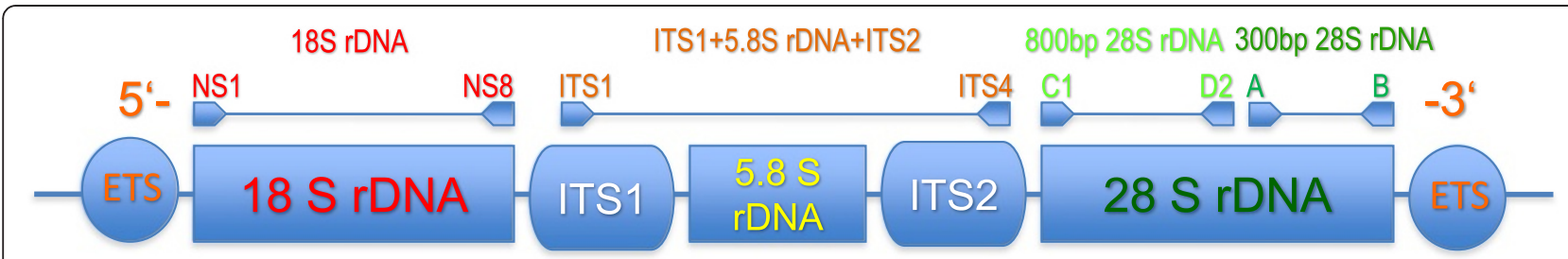

Figure 1 Schematic representation of the 45S rDNA unit and FISH probes construction including primers nesting sites. (not to scale).

karyotype category A sensu [13]. They both had 8 pairs of meta- $(\mathrm{m})$ to submetacentric $(\mathrm{sm})$ and 32 pairs of acrocentric (a) chromosomes (both sexes in C. fontanae, only males in C. albula were available), The NF was 96 in both species (Figure 2a-d). The sequential Chromomycin $\mathrm{A}_{3}\left(\mathrm{CMA}_{3}\right.$, particularly specific for $\mathrm{CG}$ rich regions) and DAPI (specific for AT rich regions) stainings revealed in both species a varying number of 6-8 sites with $\mathrm{CMA3}^{+} / \mathrm{DAPI}^{-}$signals. The signals occurred at telomeric regions of 3-4 metacentric chromosomes and at pericentromeric regions of 3-4 acrocentric/submetacentric chromosomes (Figure 3a, b). In some nuclei, several other weakly $\mathrm{CMA}_{3}^{+}$regions not corresponding to $\mathrm{DAPI}^{-}$signals mostly with pericentromeric locations were observed (Figure 3a). This variability occurs on the inter-individual as well as on the intra-individual level.

\section{Cytogenetic mapping of ribosomal DNA and comparative genomic hybridization (CGH)}

Fluorescence in situ hybridization (FISH) with $28 \mathrm{~S}$ ribosomal DNA (rDNA) probes derived from two nonoverlapping regions of the $28 \mathrm{~S}$ rRNA gene of both species (an 800 bp region adjacent towards the $5^{\prime}$-end of the $28 \mathrm{~S}$ rDNA gene and a $300 \mathrm{bp}$ region adjacent towards the 3 '-end) showed strikingly different results.
FISH using the shorter fragment as a probe revealed the presence of 6-10 chromosomes in both C. albula and C. fontanae bearing such sequences distributed similarly as the $\mathrm{CMA}_{3}^{-} / \mathrm{DAPI}^{-}$(Figure 3c for C. albula only). FISH with the longer fragment revealed bright signals on 6-10 chromosomes in C. albula (shown in colocalization with Rex1 retrotransposon, Figure 4c) but up to 40 signals (varying numbers) on chromosomes in C. fontanae (Figure 3d). Most of the signals of the $800 \mathrm{bp}$ probe of the $28 \mathrm{~S}$ rDNA in C. fontanae were localized in the AT rich (i.e. $\mathrm{DAPI}^{+}$) centromeric or pericentromeric regions of acrocentric chromosomes. Two signals of the $800 \mathrm{bp}$ $28 \mathrm{~S}$ rDNA probe corresponded to the major NOR sites evidenced also by the $300 \mathrm{bp}$ rDNA and the $\mathrm{CMA}_{3} /$ DAPI staining that were localized in telomeric regions of two large metacentric chromosomes (Figure 3d).

To verify these striking differences between $C$. albula and $C$. fontanae, we carried out a set of reciprocal comparative genomic hybridization (CGH) experiments. A mixture of the whole genome DNA (gDNA) of both C. albula and C. fontanae was hybridized simultaneously to both $C$. fontanae and C. albula chromosomes. This resulted in nearly no significant differences on $C$. albula chromosomes, i.e. a balanced hybridization of both gDNA probes was observed (Figure 3e). While signal of the $C$. fontanae gDNA when in situ compared with the a

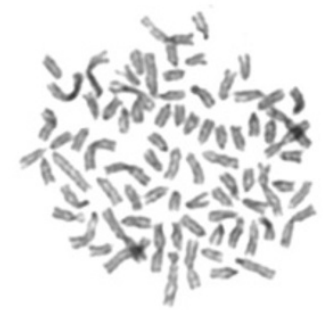

C

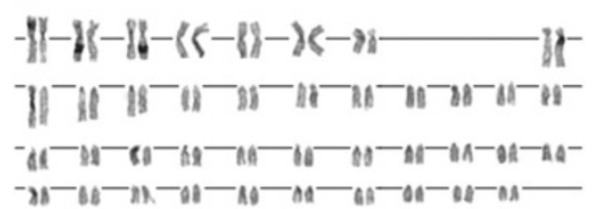

b

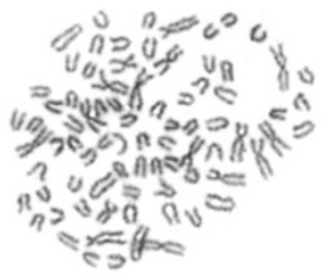

d

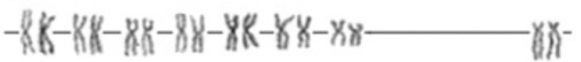

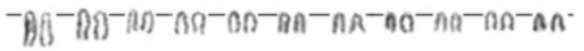

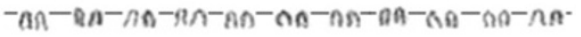

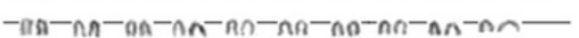

Figure 2 Giemsa-stained metaphase plates and corresponding karyogram of C. albula (a, c) and C. fontanae (b, d). Bar $=5 \mu \mathrm{m}$. 


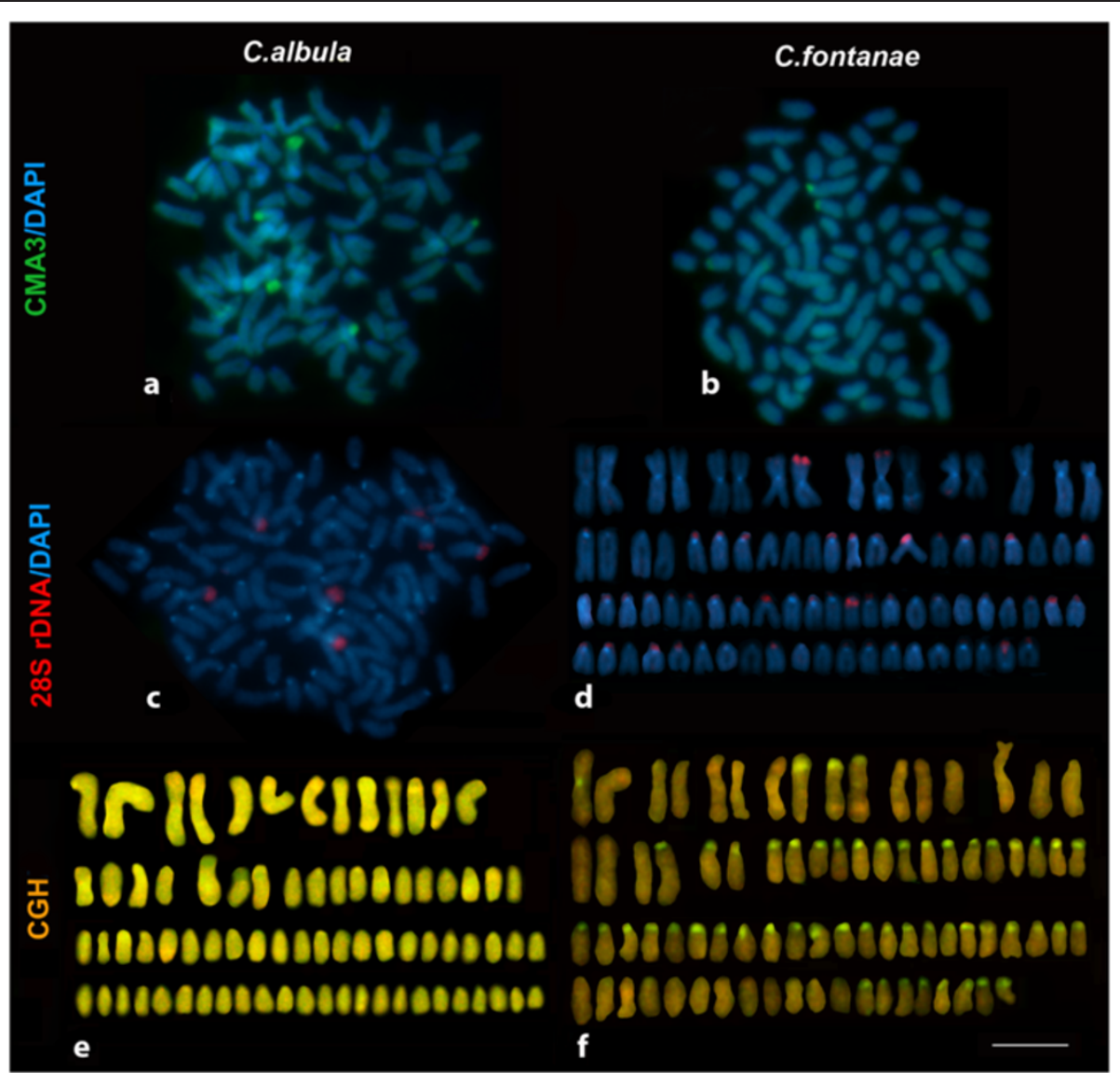

Figure 3 Metaphase plates and karyograms of $C$. albula and C. fontanae showing Chromomycin $A_{3} / D A P I$ staining, FISH and CGH experiments. Chromomycin $\mathrm{A}_{3}\left(\mathrm{CMA}_{3}\right)$ fluorescent staining (green) and DAPI (blue) staining in C. albula (a) and C. fontanae (b). FISH with the $28 \mathrm{~S}$ rDNA (300 bp probe) (red), DAPI counterstaining (blue) in C. albula (c). FISH with the 285 rDNA (800 bp probe) (red), DAPI counterstaining (blue) in C. fontanae (d). A set of reciprocal comparative genomic hybridization (CGH) experiments to C. albula chromosomes (e) and C. fontanae chromosomes (f). In both (e, f), the C. albula genomic DNA was labelled in red and the C. fontanae genomic DNA in green. Bar $=5 \mu \mathrm{m}$.

C. albula gDNA onto C. fontanae chromosomes was distinctly overrepresented in mostly pericentromeric regions of about 40 chromosomes (green signals in Figure 3f). This pattern corresponded to results of the FISH experiment with $800 \mathrm{bp} 28 \mathrm{~S}$ rDNA to C. fontanae chromosomes.

To assess quantitative differences in the distribution of the whole $45 \mathrm{~S}$ rDNA unit in both species, a further set of comparative FISH experiments with a cocktail of the $18 \mathrm{~S}$ rDNA and ITS1-ITS2 (including 5.8S rDNA) as probes amplified from both of the genomes were performed to C. albula and C. fontanae chromosomes. In the genome of $C$. albula, both the ITS1 and ITS2 were present in 6-12 signals with a varying number of signals (Figure 4a). In the genome of C. fontanae, both the ITS1 and ITS2 were multiplied to the same extent as the 800 bp $28 \mathrm{~S}$ rDNA part, i.e. a varying number of approximately 40 signals (Figure $4 \mathrm{~b}$ ). The subsequent FISH experiment with the $18 \mathrm{~S}$ rDNA in both species showed the number of 6-10 signals (Figure 4d for
C. fontanae only). The typical chromosomes bearing rDNA signals in the unamplified condition (i.e. ITS1-ITS2 and $28 \mathrm{~S}$ rDNA in $C$. albula and $18 \mathrm{~S}$ rDNA in C. fontanae) are shown in Figures $4 \mathrm{e}-\mathrm{f}$. There is a reproducible difference in location of one of the 28S rDNA in C. fontanae (when compared with C. albula) related to a distinct $\mathrm{DAPI}^{+}$band on a large metacentric chromosome pair (Figure 4f). In C. albula, the rDNA signal was always located on the opposite arm than the $\mathrm{DAPI}^{+}$band occurred (Figure 4e). In C. fontanae, one signal is located on the same arm and one signal is on the opposite one (Figure 4f). The construction of the FISH probes used in this study is visualized in Figure 1.

\section{Molecular characterization of multiplied rDNA sites}

To determine mechanisms how such extensive multiplication of parts of rRNA genes in C. fontanae might have arisen, a PCR screening for non-LTR retrotransposons in genomic DNA of both species was performed. Retrotransposons of the Rex family are known to have 


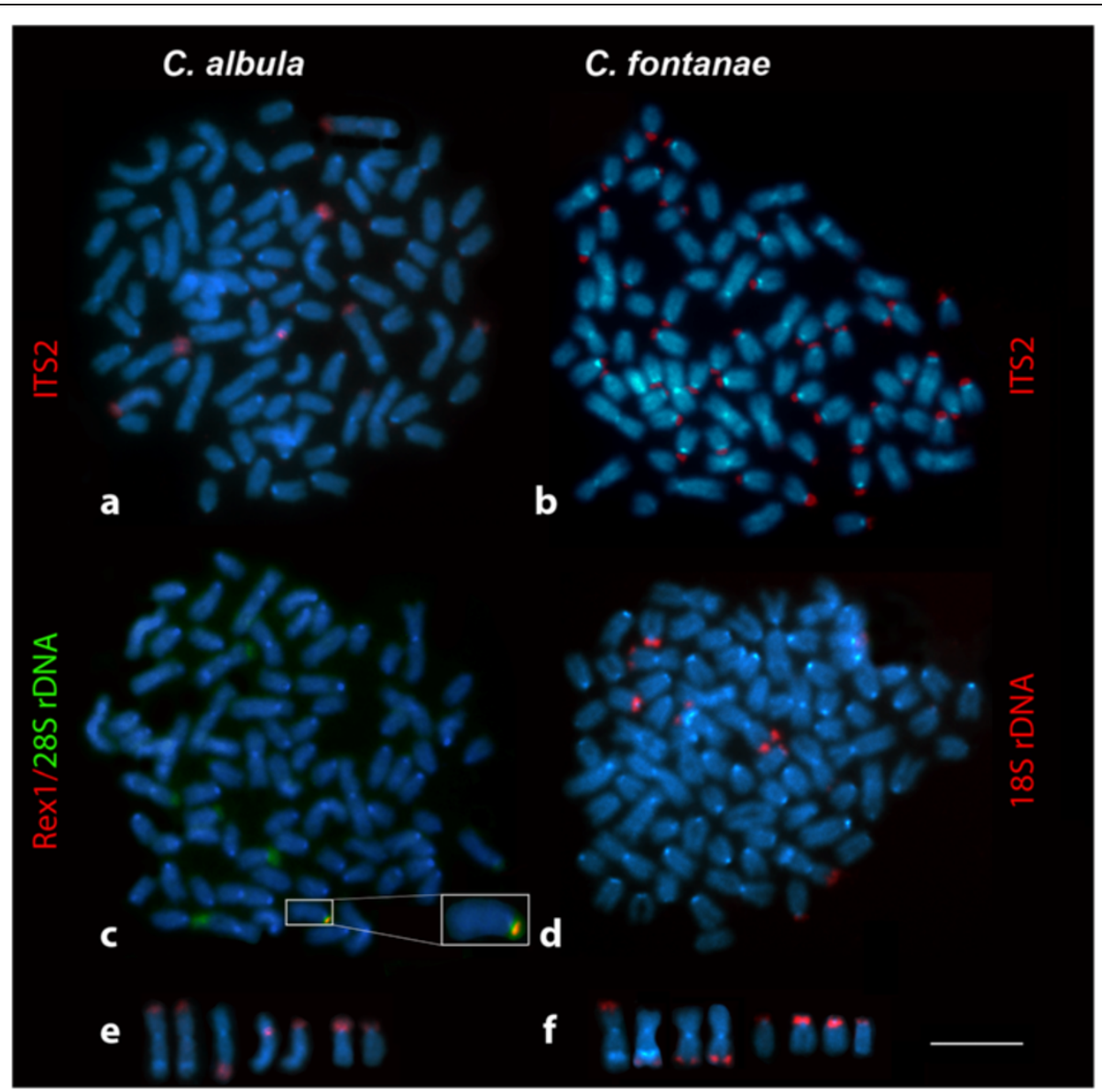

Figure 4 Metaphase plates and selected chromosomes of $C$. albula and C. fontanae showing FISH experiments. FISH with ITS2 (red) as probe hybridized to C. albula (a) and to C. fontanae (b), counterstained with DAPI. Double-FISH analysis with the Rex1 retrotransposon (red) and the 800 bp 285 rDNA (green) to C. albula (c), detail of the chromosome with Rex1 and 28S rDNA co-localization in inset. FISH with the 18S rDNA (red) to C. fontanae chromosomes (d) counterstained with DAPI. Chromosomes bearing the ITS2 (red) signal in C. albula (e). Chromosomes bearing the $18 \mathrm{~S}$ rDNA signals (red) in C. fontanae (f). Chromosomes on (f) represent the hypothetically ancestral condition of rDNA distribution prior to its multiplication in C. fontanae. Bar $=5 \mu \mathrm{m}$.

invaded fish genomes in multiple lineages [24] and to also insert into rDNA, particularly in fishes [25]. Therefore, the retroelements $\operatorname{Rex} 1, \operatorname{Rex} 3$ and $\operatorname{Rex} 6$ were tested in this study. FISH with the Rex 3 and $\operatorname{Rex} 6$ retroelements yielded inconclusive results. The Rex 1 element, as a probe hybridized to chromosomes of $C$. albula and C. fontanae, typically showed a dispersed pattern of signals on all chromosomes with a distinct accumulation in a pericentromeric region of one single acrocentric chromosome. Co-hybridization of the Rex1 element with the 800 bp $28 \mathrm{~S}$ rDNA probe in a double-FISH experiment showed co-localization of these two probes typically on one (exceptionally two to several), mostly acrocentric chromosomes in both C. albula and C. fontanae (Figure 4c for C. albula only, detail of the co-localization in inset). The Rex 1 signal with a distinctly weaker intensity occurred dispersed also on other sites corresponding to the NOR loci in both genomes. The sequences of the Rex 1 derived from the C. albula and C. fontanae genome were deposited in GenBank under the accession numbers JQ731754 and JQ731760, respectively.

Sequencing of the $18 \mathrm{~S}$ and $28 \mathrm{~S}$ rDNA, as well as ITS1 and ITS2 (deposited in GenBank under accession numbers JQ731749-JQ731753 and JQ731755-JQ731759) yielded no significant differences in these genes between C. albula and C. fontanae.

\section{Discussion}

Our findings of extensive genomic re-arrangements of a substantial fraction of the $45 \mathrm{~S} \mathrm{rDNA}$ unit in the C. fontanae genome when compared with the situation in C. albula are in strong contrast with previously reported low genetic differentiation between these two species [6,18].

Our results indicate that in the genome of $C$. fontanae next to the complete 6-10 NOR loci corresponding to similar number of NOR-bearing chromosomes in C. albula, 
up to 30 supernumerary and incomplete NOR loci occur. This is supported by results of the sequential fluorescent staining $\left(\mathrm{CMA}_{3}\right.$ and DAPI), showing about 6-8 signals in karyotypes of both species, although on chromosomes of C. fontanae the signals were slightly weaker. However, these supernumerary sites in $C$. fontanae were not represented by repeating of the complete $45 \mathrm{~S}$ rDNA unit (i.e. $18 \mathrm{~S}$ rDNA, ITS1, 5.8S rDNA, ITS2, 28S rDNA) n but only by a part including probably complete ITS1 and ITS2 and a part of the $28 \mathrm{~S}$ rDNA adjacent to the ITS2, i.e. the $5^{\prime}$ end of the $28 \mathrm{~S}$ rDNA gene (the region of $5.8 \mathrm{~S} \mathrm{rDNA}$ was not investigated separately).

Most of the supernumerary signals of the $45 \mathrm{~S}$ rDNA in chromosomes of C. fontanae were localized in the AT rich pericentromeric regions as well as the major accumulation of the Rex 1 retrotransposon on both C. albula and $C$. fontanae chromosomes. This is in accordance with findings of other authors describing accumulations of transposable elements in centromeric heterochromatin e.g. in genome of humans [26] and in a cichlid fish Cichla kelberi $[27,28]$. TEs in fishes generally tend to insert to heterochromatic areas of chromosomes ([29]; reviewed by [30]). There are also records of specific integration of some non-LTR retrotransposons at the rRNA genes found in most animal phyla (summarized by [31]), in insects Drosophila melanogaster and Bombyx mori [32] or in the fish Erythrinus erythrinus, where Rex3 retrotransposons were found in the $5 \mathrm{~S}$ rRNA genes [25].

In the above-mentioned E. erythrinus fish, a similar multiplication of rRNA genes was described [25]. In that case, four karyomorphs of E. erythrinus differ in their chromosomal number, karyotype, presence or absence of heteromorphic sex chromosomes and numbers of $5 \mathrm{~S}$ rDNA loci. The karyomorph A in E. erythrinus showed only two $5 \mathrm{~S}$ rDNA loci, while in the karyomorph $\mathrm{D}$, 21-22 5S rDNA loci could be observed. All 5S rDNA sites co-localized with the Rex3 retrotransposon. On the other hand, no changes in the heterochromatin and $18 \mathrm{~S}$ rDNA patterns were found between these two karyomorphs [25]. Such two karyomorphs within a single species $E$. erythrinus may be seen as an incipient stage of a speciation event. This situation can thus represent an initial stage, later resulting in the condition observed in morphologically [15], ecologically and physiologically $[33,34]$ diverged species pair C. fontanae and C. albula described in this study. A similar observation of extremely multiplied NOR sites (46 and 49 countable FISH signals), however, without any further detailed analysis, were reported in brook char Salvelinus fontinalis (Salmonidae) [35].

In salmonid fishes, TEs have been studied intensively [30,36,37]. Microarray studies showed that transcription of rainbow trout transposons is activated by external stimuli, such as toxicity, stress and bacterial antigens [38]. In the oligotrophic Lake Stechlin, the food availability for coregonines was extremely limited and the size at maturity and the maximal size of $C$. albula are far behind the other populations of this species in adjacent lakes in northern Germany [39]. C. fontanae is the smallest species of the genus Coregonus in Europe [8]. Raising both species in the laboratory demonstrated that both grew much larger if supported with unlimited food (unpublished obs., Freyhof). Therefore, it can be speculated that both species, especially C. fontanae, live in an extreme permanent starvation in the Lake Stechlin. It can be also hypothesized that the springspawning habit of $C$. fontanae might have originated simply by the shift of sexual maturity in the part of the population that has not been able to attain sexual maturity in autumn due to the lower food intake and hence environmental starvation stress.

\section{Link between environmental stress and chromatin modification/regulation}

Effects of stress on the genome can result in important perturbations creating new combinations better compatible with survival (summarized by [40]; more recently reviewed by [41]). After the discovery of transposable elements (TE) more than 50 years ago, their mutagenic effect had been increasingly viewed in association with rapid genome reorganizations by the creation of new regulation patterns and chromosome restructuring during last years [41]. Stress activated mobilization of these elements by failure of epigenetic silencing (the host defence model of repressing the movement of mobile elements; [42,43]) can lead to (re)activation of mobile elements and consequently to major and rapid genome alterations $[40,41,44,45]$.

Barbara McClintock [46] already considered TE as a source of hypermutagenicity creating viable and fertile individuals from a stressed population under risk of extinction. Moreover, she originally named TE "controlling elements" due to their ability to alter gene activity and genome structure [47].

\section{TE-mediated genome rearrangements as a factor in speciation}

With growing evidence for the importance of TEs in the genome evolution, the role of TE-mediated genome changes in the speciation by their possible contribution to pre- and post-mating reproductive isolation formation has been increasingly taken into account and discussed generally in eukaryotes [48,49], Drosophila [50], fishes [51], mammals [52], and plants [53]. However, lack of experimental data makes it difficult to prove this possibility (reviewed by $[51,54,55]$ ). On the other hand, [41] provides an overview of TE transposition bursts 
concomitant with radiation periods in seven cases. The same authors also discuss TE-induced rapid speciation associated with the ability of TEs to induce chromosomal rearrangements. Therefore, the sympatric species pair C. albula and C. fontanae in the context of other congeneric coregonine species and their variable evolutionary history in the Eurasian post-glacial lakes appears to be a suitable model system for exploring mechanisms of genomic differentiation and speciation with or without TE contribution.

In a very similar, but North American study system (lake whitefish species pairs, Coregonus spp.), [56] next generation sequencing (NGS) showed that TEs appeared to be highly expressed in hybrids between two recently diverged species. This may be potentially the mechanism responsible for post-zygotic reproductive isolation. Moreover, NGS can be viewed as a useful tool complementary with molecular cytogenetic approach presented in this study enabling confirmation of here documented results and search for other candidate groups of TEs involved in the genome re-arrangements and accelerated speciation.

\section{Conclusion}

In the sympatric species pair C. albula and C. fontanae, we encounter a complex situation involving several evolutionary phenomena and factors. Firstly, a rapid ecological speciation event with an unclear sympatric scenario, i.e. the derived species $C$. fontanae fully differentiated from $C$. albula physiologically, ecologically and morphologically within about $12-14$ kyrs in the newly colonized Stechlin Lake after the last glacier retreated [15]. Secondly, genetic differentiation of these two species remained weak as the combined analyses of mtDNA and microsatellite loci [18] showed, as well as major karyotypic and chromosomal markers presented in this study. This is in contrast with extensive genome re-arrangements in a large proportion of the $45 \mathrm{~S}$ rDNA cassette in $C$. fontanae when compared with its most likely ancestral species - C. albula. The genome rearrangements are exhibited as a distinct loci number differences and relocation of variable number (about 30) AT rich pericentromeric regions in C. fontanae. The molecular mechanism behind these re-arrangements might be a retrotransposition of a part of the $45 \mathrm{~S}$ rDNA unit mediated by retrotransposons. Retrotransposonal activity can be mobilized under certain conditions (stress, environmental changes) and cause rapid and extensive structural changes to the host genome. These structural genomic differences in $C$. fontanae accumulated to pericentromeric heterochromatin in almost half of the chromosome complement. This might then have been acting as a partial but permanent reproductive barrier by hampering recombination, thus, enabling and accelerating the morphological, ecological and physiological differentiation of C. fontanae. Moreover, interspecific hybridization between the old and the newly arising species might have activated retrotransposonal activity in hybrids resulting in hybrid sterility or unviability as reviewed by [51]. The population genetic parameters of this speciation event, favouring fixation of the re-arranged genomes, remain to be elucidated in detail, but small effective population size is a good hypothesis to be tested.

\section{Methods}

\section{Materials}

For this study, we had 12 individuals of Coregonus albula (Linnaeus, 1758) and 16 individuals of C. fontanae [15], both from Lake Stechlin (northern Germany, Brandenburg, $53^{\circ} 10^{\prime} \mathrm{N} ; 13^{\circ} 02^{\prime} \mathrm{E}$ ). All fish were raised in the laboratory under identical conditions as described by [33,34]. In C. albula, 3 individuals (samples alb 1, 2 and 5, males only) yielded metaphases usable for down-stream FISH and CGH experiments. In C. fontanae, 3 individuals also (samples font 2, 5 and 7, both males and females) yielded usable chromosome preparations. Of all studied individuals, we isolated genomic DNA from fin clips and muscles. All tissue and DNA samples, including cell suspensions and chromosome preparations, are deposited in the Laboratory of Fish Genetics of the Institute of Animal Physiology and Genetics (IAPG). This study was covered by the "Valid Animal Use Protocols" Nr. CZ 00221 at the IAPG issued by the Czech Ministry of Agriculture on 10 June 2009.

\section{Chromosome preparations}

Metaphases were prepared according to [57] with slight modifications. Briefly, the fish were injected with $0.1 \%$ colchicine solution (w/v, SIGMA), $1 \mathrm{ml} / 100 \mathrm{~g}$ body weight, for 45 minutes then sacrificed by overdose of anaesthetic $0.5 \%$ Phenoxyethanol (v/v, SIGMA). Kidneys were removed, dissected in $0.075 \mathrm{M} \mathrm{KCl}$ and the cell suspension free of tissue fragments was hypotonized for $8 \mathrm{~min}$ in $0.075 \mathrm{M} \mathrm{KCl}$, fixed in methanol: acetic acid 3:1 (v/v) fixative, washed twice in fixative, and finally spread onto slides (Superfrost quality). Mitotic activity was not stimulated because these fish showed extremely high sensitivity to agents increasing mitotic rate. Simultaneously, the blood (around $0.5 \mathrm{ml}$ ) was collected from all analysed individuals by fine heparinized syringe for leukocyte culture according to the protocol of [58]. Briefly, partly washed leukocytes were cultivated in $5 \mathrm{ml}$ of a complete medium composed of TC 199 (SIGMA, St. Louis, MO, USA), 10\% FBS Superior (Biochrom, Berlin, Germany), 0.5\% Antibiotic Antimycotic Solution (SIGMA), 1\% Kanamycin monosulfate (SIGMA), 1\% LPS (SIGMA), 0.2\% PHA H15 (Remel, Lenexa, KS, USA) and 0.175ųl Mercaptoethanol (SIGMA) at $19.5^{\circ} \mathrm{C}$ for 6-7 days, 
then 2 drops of the $0.1 \%$ colchicine were added for 45 minutes at RT and cells harvested as for the direct preparation described above.

\section{Fluorescence in situ hybridization (FISH) and comparative} genomic hybridization (CGH)

Probes for in situ hybridization experiments were produced either by PCR (FISH probes) or directly from the genomic DNA (CGH probes). Probes were indirectly labelled with haptens (biotin and digoxigenin) by means of nick translation (whole genomic DNA and FISH probe longer than $600 \mathrm{bp}$ ) using the Roche Nick Translation Mix (Roche, Mannheim, Germany; Cat.No. 11745808910) according to the manufacturer's instructions. Shorter DNA fragments were labelled by PCR using the Roche PCR DIG Labeling Mix (Cat.No. 11585550910). The biotin-dUTP labelled probes (Roche, Cat. No. 11093070910) were detected by either the Invitrogen $\mathrm{Cy}^{\mathrm{TM}}$ 3-Streptavidin (Invitrogen, San Diego, CA, USA;
Cat.No. 43-4315) or by the FITC-Streptavidin (Cat.No. 43-4311). The digoxigenin-dUTP labelled probes (Roche, Cat.No. 11093088910) were detected by either the Roche Anti-Digoxogenin-Fluorescein (Cat.No. 11207741910) or by the Anti-Digoxigenin-Rhodamin (Cat.No. 11207750910). An unlabelled DNA competitor for suppression of nonspecific hybridization of fragment size of 100-200 bp was added with 20-fold the concentration of the DNA probe in CGH experiments. The CGH DNA probe concentration was 1 ug per reaction for both genomes compared. An aging of chromosome preparations at $37^{\circ} \mathrm{C}$ for 3 hours was carried out prior to each of the hybridization experiment. Pepsinization, hybridization and detection were carried out under conditions as described by [59].

All rDNA FISH probes were constructed using published, mostly generally used PCR primer sets of the $45 \mathrm{~S}$ rDNA unit to cover its major regions and to map them physically onto chromosomes.

Table 1 PCR primers used in this study

\begin{tabular}{|c|c|c|c|}
\hline Name & Region of DNA/FISH probe & Primer sequence ( $5^{\prime}$ to $3^{\prime}$ ) & Ref. \\
\hline $28 \mathrm{~S} A$ & $3^{\prime}$ end of the 285 rDNA involving the regions $A$ and $B, F$ primer & AAA CTC TGG TGG AGG TCC GT & [61] \\
\hline $28 \mathrm{~S} B$ & Internally nested in the regions $A$ and $B$ of the 285 rDNA, R primer & CTT ACC AAA AGT GGC CCA CTA & [61] \\
\hline $28 \mathrm{SC1}$ & $5^{\prime}$ end of the $28 \mathrm{~S}$ rDNA adjacent to the ITS2, F primer & ACC CGC TGA ATT TAA GCA T & [62] \\
\hline 28S D2 & Internally nested in the region C3 involving D2, C2, D1, C1, R primer & TCC GTG TIT CAA GAC GGG & [63] \\
\hline ITS1 & $3^{\prime}$ end of the 185 rDNA adjacent to the ITS1, F primer & TCC GTA GGT GAA CCT GCG G & [64] \\
\hline ITS2 & $3^{\prime}$ end of the $5.8 \mathrm{~S}$ rDNA adjacent to the ITS2, R primer & GCT GCG TTC TTC ATC GAT GC & [64] \\
\hline ITS3 & $5^{\prime}$ end of the $5.8 \mathrm{~S}$ rDNA adjacent to the ITS1, F primer & GCA TCG ATG AAG AAC GCA GC & [64] \\
\hline ITS4 & $5^{\prime}$ end of the $28 \mathrm{~S}$ rDNA adjacent to the ITS2, R primer & TCC TCC GCT TAT TGA TAT GC & [64] \\
\hline NS1 & $5^{\prime}$ end of the 185 rDNA F primer & GTA GTC ATA TGC TTG TCT & [64] \\
\hline NS2 & $18 \mathrm{~S}$ rDNA R primer & GGC TGC TGG CAC CAG ACT TGC & [64] \\
\hline NS3 & $18 \mathrm{~S}$ rDNA F primer & GCA AGT CTG GTG CCA GCA GCC & [64] \\
\hline NS4 & $18 \mathrm{~S}$ rDNA R primer & CTT CCG TCA ATT CCT TTA AG & [64] \\
\hline NS5 & $18 \mathrm{~S}$ rDNA F primer & AAC TTA AAG GAA TTG ACG GAA G & [64] \\
\hline NS6 & $18 \mathrm{~S}$ rDNA R primer & GCA TCA CAG ACC TGT TAT TGC CTC & [64] \\
\hline NS7 & $18 \mathrm{~S}$ rDNA F primer & GAG GCA ATA ACA GGT CTG TGA TGC & [64] \\
\hline NS8 & 3 ' end of the 185 rDNA R primer & TCC GCA GGT TCA CCT ACG GA & [64] \\
\hline RTX1F1 & Rex1 F primer & TTC TCC AGT GCC TTC AAC ACC & [28] \\
\hline RTX1R3 & Rex1 R primer & TCC CTC AGC AGA AAG AGT CTG CTC & {$[28]$} \\
\hline RTX3F1 & Rex3 F primer & TAC GGA GAA AAC CCA TाT CG & [65] \\
\hline RTX3F2 & Rex3 F primer & AAC ACC TTG GCT GCG CCT AG & [65] \\
\hline RTX3F3 & Rex3 F primer & CGG TGA YAA AGG GCA GCC CTG & [28] \\
\hline RTX3R1 & Rex3 R primer & AAA GTT CCT CGG TGG CAA GG & [65] \\
\hline RTX3R2 & Rex3 R primer & CCR GGG GTG GAT GAR RTC CGC CC & [65] \\
\hline RTX3R3 & Rex3 R primer & TGG CAG ACN GGG GTG GTG GT & {$[28]$} \\
\hline RTX6F & Rex6 F primer & TAA AGC ATA CAT GGA GCG CCA C & {$[28]$} \\
\hline RTX6R & Rex6 R primer & GGT CCT CTA CCA GAG GCC TGG G & [28] \\
\hline
\end{tabular}




\section{PCR amplification of FISH probes and the analysis of the 45S rDNA unit}

All primer sets used in this study are summarized in Table 1. Primers nesting within the 45S rDNA unit relevant for this study are shown in Figure 1. Thermal profiles were used according to references given in Table 1. FISH probes were constructed from PCR conducted on the respective species as they were later hybridized. All sequences used in this study as FISH probe or in the molecular-biological analyses of the $45 \mathrm{~S}$ rDNA unit were deposited in the GenBank [60] under accession numbers JQ731749 - JQ731760.

\section{Cloning, sequencing and sequences analysis}

PCR products were cloned using the QIAGEN PCR Cloning Kit and QIAGEN EZ Competent Cells (Qiagen, Hilden, Germany); the plasmids were isolated from the cells with Qia PREP Spin Miniprep Kit according to the manufacturer's instructions. The primary PCR products were first sequenced on the ABI 3130 Genetic Analyzer (Applied Biosystems, Hitachi, Foster City, CA, USA) using the BigDye Terminator Cycle Sequencing Kit (Applied Biosystems). Furthermore, cloned DNA fragments that were later applied as FISH probes were commercially sequenced by Macrogen (Seoul, South Korea). The commercially obtained sequences were subjected to online megablast or discontiguous megablast [66] searches at the National Center for Biotechnology Information (NCBI) [67], where their similarity to the sequences deposited in the GenBank databases was checked.

\section{Microscopy and image processing}

Chromosome preparations were analysed with the Provis AX70 Olympus microscope equipped with standard fluorescence filter sets. Gray-scale hybridization signals on chromosomes and/or DAPI counterstained chromosomes were captured by the CCD camera (DP30W Olympus). Using the Olympus Acquisition Software, black and white images were pseudo-coloured and superimposed with the software MicroImage. The colour images have been analyzed and processed with Adobe Photoshop, Version CS5. The chromosomes were classified using the nomenclature proposed by [68]. Karyotypes based on the Giemsa-stained chromosomes were produced using the IKAROS (Metasystems) software. Chromosomal formulas were formed according to [69].

\section{Abbreviations}

AFLP: Amplified fragment length polymorphism; CGH: Comparative genomic hybridization; $\mathrm{CMA}_{3}$ : Chromomycin $\mathrm{A}_{3}$; DAPI: 4', 6-diamidino-2-phenylindole; DAPI/CMA ${ }_{3}^{+/-}$: DAPI/CMA 3 positive/negative signals; FISH: Fluorescence in situ hybridization; IAPG: Institute of animal physiology and genetics; kyrs BP: Thousand years before presence; NF: "Nombre fundamental" chromosome arm number; NGS: Next generation sequencing; NOR: Nucleolar organizer DNA; rDNA: Ribosomal DNA; TE: Transposable elements; 2n: Diploid chromosome number.
Competing interests

There are no competing interests to declare.

\section{Authors' contributions}

RS designed and performed experiments FISH and CGH and co-drafted the manuscript, ZM and AS performed FISH experiments, ZM performed PCRs and sequenced PCR products and co-drafted the manuscript, GS and JF collected and raised material, JB and RS prepared chromosome preparations, MR partly contributed to digital processing of images, PR designed the study and co-drafted the manuscript. All authors read and approved the final manuscript.

\section{Acknowledgements}

This study was supported in parts by the projects No. 523/08/0824 and No. P506/11/P596 of the Grant Agency of the Czech Republic, No. LC06073 Biodiversity Research of the Ministry of Education, Youth and Sports of the Czech Republic and No. IRP IAPG AVOZ 50450515

This study is a part of the series "Chromosome evolution in Salmonidae".

\section{Author details}

${ }^{1}$ Laboratory of Fish Genetics, Institute of Animal Physiology and Genetics, Czech Academy of Sciences, Rumburská 89, Liběchov 277 21, Czech Republic. ${ }^{2}$ Leibniz-Institut of Freshwater Biology and Inland Fisheries, Müggelseedamm 310, Berlin 12587, Germany.

Received: 29 May 2012 Accepted: 11 February 2013

Published: 14 February 2013

\section{References}

1. Cristescu ME, Adamowicz SJ, Vaillant JJ, Haffner DG: Ancient lakes revisited: from the ecology to the genetics of speciation. Mol Ecol 2010, 19:4837-4851

2. Schluter D: Ecological speciation in postglacial fishes. Philos T Roy Soc B 1996, 351:807-814

3. Nelson JS: Fishes of the world. 4th edition. Hoboken, NJ: John Wiley \& Sons; 2006.

4. Bodaly RA, Vuorinen J, Ward RD, Luczynski M, Reist JD: Genetic comparison of new and old world coregonid fishes. J Fish Biol 1991, 38:37-51.

5. Hudson $A G$, Vonlanthen $P$, Seehausen $O$ : Rapid parallel adaptive radiations from a single hybridogenetic ancestral population. P Roy Soc B-Biol 2011, 278:58-66.

6. Mehner T, Pohlmann K, Elkin C, Monaghan MT, Nitz B, Freyhof J: Genetic population structure of sympatric and allopatric populations of Baltic ciscoes (coregonus albula complex, teleostei, coregonidae). BMC Evol Biol 2010, 10:85.

7. Douglas MR, Brunner PC, Bernatchez L: Do assemblages of coregonus (teleostei: salmoniformes) in the central alpine region of Europe represent species flocks? Mol Ecol 1999, 8:589-603.

8. Kottelat M, Jörg F: Handbook of European Freshwater Fishes. Cornol, Switzerland: Publications Kottelat; 2007.

9. Vonlanthen $P$, Roy D, Hudson AG, Largiader CR, Bittner D, Seehausen O: Divergence along a steep ecological gradient in lake whitefish (coregonus sp.). J Evolution Biol 2009, 22:498-514.

10. Taylor EB: Species pairs of north temperate freshwater fishes: evolution, taxonomy, and conservation. Rev Fish Biol Fisher 1999, 9:299-324.

11. Vuorinen J, Himberg M, Lankinen P: Genetic differentiation in coregonus albula (salmonidae) populations in Finland. Hereditas 1981, 94:113-121.

12. Hudson AG, Vonlanthen $P$, Müller R, Seehausen O: Review: the geography of speciation and adaptive radiation in coregonines. Adv Limnol 2007, 60:111-146

13. Phillips R, Ráb P: Chromosome evolution in the salmonidae (pisces): an update. Biol Rev 2001, 76:1-25.

14. Quimseyh MB: Evolution of number and morphology of mammalian chromosomes. J Hered 1994, 85:455-465.

15. Schulz M, Freyhof J: Coregonus fontanae, a new spring-spawning Cisco from lake stechlin, northern Germany (salmoniformes: coregonidae). Ichthyol Explor Fresh 2003, 14:209-216.

16. Ohlberger J, Mehner T, Staaks G, Hoelke F: Is ecological segregation in a pair of sympatric coregonines supported by divergent feeding efficiencies? Can J Fish Aquat Sci 2008, 65:2105-2113. 
17. Jankun M, Martinez P, Pardo BG, Kirtiklis L, Rab P, Rabova M, Sanchez L: Ribosomal genes in coregonid fishes (coregonus lavaretus, C. Albula and C. Peled) (salmonidae): single and multiple nucleolus organizer regions. Heredity 2001, 87:672-679.

18. Schulz M, Freyhof J, Saint-Laurent R, Østbye K, Mehner T, Bernatchez L: Evidence for independent origin of two spring-spawning ciscoes (Salmoniformes: Coregonidae) in Germany. J Fish Biol 2006, 68:119-135.

19. Swarzacher HG, Wachtler F: The nucleolus. Anat Embryol 1993, 188:515-536.

20. Wachtler F, Stahl A: The nucleolus: a structural and functional interpretation. Micron 1993, 24:473-505.

21. Moss T, Stefanovsky VY: Promotion and regulation of ribosomal transcription in eukaryotes by RNA polymerase I. Prog Nucleic Acids Res Mol Biol 1994, 50:25-66.

22. Wang S, Zhao M, Li T: Complete sequence of the $10.3 \mathrm{~kb}$ silkworm attacus ricini rDNA repeat,determination of the transcriptional initiation site and functional analysis of the intergenic spacer. DNA Seq 2003, 14:95-101.

23. Hillis DM, Dixon MT: Ribosomal DNA: molecular evolution and phylogenetic inference. Q Rev Biol 1991, 66:411-453.

24. Volff J-N, Körting C, Schartl M: Multiple lineages of the non-LTR retrotransposon Rex 1 with varying success in invading fish genomes. Mol Biol Evol 2000, 17:1673-1684.

25. Cioffi MB, Martins C, Bertollo LA: Chromosome spreading of associated transposable elements and ribosomal DNA in the fish Erythrinus erythrinus. Implications for genome change and karyoevolution in fish. BMC Evol Biol 2010, 10:271.

26. Wong $\mathrm{LH}$, Andy Choo $\mathrm{KH}$ : Evolutionary dynamics of transposable elements at the centromere. Trends Genet 2004, 20:611-616.

27. Teixeira WG, Ferreira IA, Cabral-de-Mello DC, Mazzuchelli J, Valente GT, Pinhal D, Poletto AB, Martins C: Organization of repeated DNA elements in the genome of the cichlid fish cichla kelberi and its contribution to the knowledge of fish genomes. Cytogenet Genome Res 2009, 125:224-234.

28. Valente GT, Mazzuchelli J, Ferreira IA, Poletto AB, Fantinatti BEA Cytogenetic mapping of the retroelements Rex1, Rex3 and Rex6 among cichlid fish: new insights on the chromosomal distribution of transposable elements. Cytogenet Genome Res 2011, 133:34-42

29. DaSilva C, Hadji H, Ozouf-Costaz C, Nicaud S, Jaillon O, Weissenbach J, Roest Crollius H: Remarkable compartmentalization of transposable elemetns and pseudogenes in the heterochromatin of the tetraodon nigroviridis genome. Proc Natl Acad Sci U S A 2002, 99:1636-1641.

30. Koop BF, Davidson WS: Genomics and the Genome Duplication in Salmonids. In Fisheries for Global Welfare and Environment. Edited by Tsukamoto K, Kawamura T, Takeuchi T, Beard TD Jr, Kaiser MJ. Tokyo: Terrapub; 2008:77-86.

31. Kurzynska-Kokorniak A, Jamburuthugoda VK, Bibillo A, Eickbush TH: DNA directed DNA polymerase and strand displacement activity of the reverse transcriptase encoded by the R2 retrotransposon. J Mol Biol 2007, 374:322-333.

32. Jakubczak JL, Xiong Y, Eickbush TH: Type I (RI) and type II (R2) ribosomal DNA insertions of drosophila melanogaster are retrotransposable elements close11 related to those of bombyx Mori. J Mol Biol 1990, 212:37-52.

33. Ohlberger J, Mehner T, Staaks G, Hölker F: Temperature-related physiological adaptations promote ecological divergence in a sympatric species pair of temperate freshwater fish. Coregonus spp. Funct Ecol 2008, 22:501-508.

34. Ohlberger J, Staaks G, Petzoldt T, Mehner T, Hölker F: Physiological specialization by thermal adaptation drives ecological divergence in a sympatric fish species pair. Evol Ecol Res 2008, 10:1173-1185.

35. Fujiwara A, Abe S, Yamaha E, Yamazaki F, Yoshida MC: Chromosomal localization and heterochromatin association of ribosomal RNA gene loci and silver-stained nucleolar organizer regions in salmonid fishes. Chromosome Res 1998, 6:463-471.

36. Matveev V, Okadaa N: Retroposons of salmonoid fishes (actinopterygii: salmonoidei) and their evolution. Gene 2008, 434:16-28.

37. De Boer JG, Yazawa R, Davidson WS, Koop BF: Burst and horizontal evolution of DNA transposons in the speciation of pseudotetraploid salmonids. BMC Genomics 2007, 8:422
38. Krasnov A, Koskinen H, Afanasyev A, Mölsä H: Transcribed Tcl-like transposons in salmonid fish. BMC Genomics 2005, 6:107.

39. Helland IP, Harrod C, Freyhof J, Mehner T: Co-existence of a pair of pelagic planktivorous coregonid fishes. Evol Ecol Res 2008, 10:373-390

40. Arnault C, Dufournel I: Genome and stresses: reactions against aggressions, behaviour of transposable elements. Genetica 1994 93:149-160.

41. Rebollo R, Horard B, Hubert B, Vieira C: Jumping genes and epigenetics: towards new species. Gene 2010, 454:1-7.

42. Bestor TH, Tycko B: Creation of genomic methylation patterns. Nat Genet 1996, 12:363-367.

43. Yoder JA, Walsh CP, Bestor TH: Cytosine methylation and the ecology of intragenomic parasites. Trends Genet 1997, 13:335-340.

44. Wichman HA, Van Den Bussche RA, Hamilton MJ, Baker RJ: Transposable elements and the evolution of genome organization in mammals. Genetica 1992, 86:287-293.

45. Biémont C: A brief history of the status of transposable elements: from junk DNA to major players in evolution. Genetics 2010, 186:1085-1093.

46. McClintock B: The significance of responses of the genome to challenge. Science 1984, 226:792-801.

47. McClintock B: Mutable loci in maize. Carnegie Inst Wash Yrbk 1949. 48:142-154

48. Hurst GDD, Werren $\mathrm{JH}$ : The role of selfish elements in eukaryotic evolution. Nat Rev Genet 2001, 2:597-606.

49. Hua-Van A, Le Rouzic A, Boutin TS, Filée J, Capy P: The struggle for life of the genome's selfish architects. Biol Direct 2011, 6:19.

50. Vieira C, Lepetit D, Dumont S, Biémont C: Wake up of transposable elements following drosophila simulans worldwide colonization. Mol BiO Evol 1999, 16:1251-1255.

51. Volff JN: Genome evolution and biodiversity in teleost fish. Heredity 2005 94:280-294.

52. Böhne A, Brunet F, Galiana-Arnoux D, Schultheis C, Volff J-N: Transposable elements as drivers of genomic and biological diversity in vertebrates. Chromosome Res 2008, 16:203-215.

53. Noor MAF, Chang AS: Evolutionary genetics: jumping into a New species. Curr Biol 2006, 16:R890-R892.

54. Hurst GDD, Schilthuizen M: Selfish genetic elements and speciation. Heredity 1998, 80:2-8.

55. Kazazian HH Jr: Mobile elements: drivers of genome evolution. Science 2004, 303:1626-1632.

56. Renaut S, Nolte AW, Bernatchez L: Mining transcriptome sequences towards identifying adaptive single nucleotide polymorphisms in lake whitefish species pairs (coregonus spp. Salmonidae). Mol Ecol 2010, 19(Suppl.1):115-131.

57. Ráb P, Roth P: Cold-blooded vertebrates. In Methods of chromosome analysis. Edited by Balicek P, Forejt J, Rubes J. Brno: Cytogenet Sect Cs Biol Soc Publishers; 1988:115-124.

58. Fujiwara A, Nishida-Umehara C, Sakamoto T, Okamoto N, Nakayama I, Abe S: Improved fish lymphocyte culture for chromosome preparation. Genetica 2001, 111:77-89.

59. Cremer M, Grasser F, Lanctôt C, Müller S, Neusser M, Zinner R, Solovei I, Cremer T: Multicolor 3D Fluorescence In Situ Hybridization for Imaging Interphase Chromosomes. In The Nucleus: Volume I: Nuclei and Subnuclear Components, Methods in Molecular Biology ${ }^{T M}$, Volume Chapter 15, Volume 463. Edited by Hancock R.: Humana Press; 2008:205-239. Springer Protocols.

60. Database GenBank. http://www.ncbi.nlm.nih.gov/genbank/.

61. Zhang Q, Cooper RK, Tiersch TR: Chromosomal location of the $28 \mathrm{~S}$ ribosomal RNA gene of channel catfish by in situ polymerase chain reaction. J Fish Biol 2000, 56:388-397

62. Dayrat B, Tillier A, Lecointre G, Tillier S: New clades of euthyneuran gastropods (mollusca) from $28 \mathrm{~S}$ rRNA sequences. Mol Phylogenet Evol 2001, 19:225-235.

63. Chombard C, Boury-Esnault N, Tillier S: Reassessment of homology of morphological characters in tetractinellid sponges based on molecular data. Syst Biol 1998, 47:351-366.

64. White TJ, Bruns T, Lee S, Taylor JW: Amplification and direct sequencing of fungal ribosomal RNA genes for phylogenetics. In PCR Protocols: A Guide to Methods and Applications. Edited by Innis MA, Gelfand DH, Sninsky JJ, White TJ. New York: Academic Press Inc; 1990:315-322. 
65. Volff JN, Körting C, Meyer A, Schartl M: Evolution and discontinuous distribution of Rex3 retrotransposons in fish. Mol Biol Evol 2001, 18:427-431

66. Altschul SF, Gish W, Miller W, Myers EW, Lipman DJ: Basic local alignment search tool. J Mol Biol 1990, 215:403-410.

67. National Center for Biotechnology Information. http://www.ncbi.nlm.nih.gov/ blast.

68. Levan AK, Fredga K, Sandberg AA: Nomenclature for centromeric position on chromosomes. Hereditas 1964, 52:201-220.

69. Matthey R: L'evolution de la formule chromosomale chez les vertébrés. Experientia 1945, 1:50-56

doi:10.1186/1471-2148-13-42

Cite this article as: Symonová et al:: Genome differentiation in a species pair of coregonine fishes: an extremely rapid speciation driven by stress-activated retrotransposons mediating extensive ribosomal DNA multiplications. BMC Evolutionary Biology 2013 13:42.

\section{Submit your next manuscript to BioMed Central and take full advantage of:}

- Convenient online submission

- Thorough peer review

- No space constraints or color figure charges

- Immediate publication on acceptance

- Inclusion in PubMed, CAS, Scopus and Google Scholar

- Research which is freely available for redistribution 